\title{
A General Model to Optimise Cu" Labelling Efficiency of Double-Histidine Motifs for Pulse Dipolar EPR Applications
}

Joshua L. Wort, ${ }^{a}$ Katrin Ackermann, ${ }^{a}$ David G. Norman, ${ }^{b}$ and Bela E. Bode*a

(a) EaStCHEM School of Chemistry, Biomedical Sciences Research Complex, and Centre of Magnetic Resonance, University of St Andrews North Haugh, St Andrews KY16 9ST (UK)

(b) School of Life Sciences, University of Dundee, Medical Sciences Institute, Dundee, DD1 $5 \mathrm{EH}(\mathrm{UK})$

\begin{abstract}
Electron paramagnetic resonance (EPR) distance measurements are making increasingly important contributions to studies of biomolecules underpinning health and disease by providing highly accurate and precise geometric constraints. Combining double-histidine $(\mathrm{dH})$ motifs with $\mathrm{Cu}$ "l spin labels shows promise for further increasing the precision of distance measurements, and for investigating subtle conformational changes. However, non-covalent coordination-based spin labelling is vulnerable to low binding affinity. Dissociation constants of $\mathrm{dH}$ motifs for $\mathrm{Cu}^{\prime \prime}$-nitrilotriacetic acid were previously investigated via relaxation induced dipolar modulation enhancement (RIDME), and demonstrated the feasibility of exploiting the double histidine motif for EPR applications at sub- $\mu$ M protein concentrations. Herein, the feasibility of using modulation depth quantitation in Cu"-Cu" RIDME to simultaneously estimate a pair of non-identical independent $K_{D}$ values in such a tetra-histidine model protein is addressed. Furthermore, we develop a general speciation model to optimise Cull labelling efficiency, in dependence of pairs of identical or disparate $K_{D}$ values and total $\mathrm{Cu}^{\prime \prime}$ label concentration. We find the dissociation constant estimates are in excellent agreement with previously determined values, and empirical modulation depths support the proposed model.
\end{abstract}

\section{Introduction}

Pulse dipolar electron paramagnetic resonance (PDEPR) spectroscopy is an attractive methodology to supplement crystallography, FRET, cryo-EM, or NMR data with nanometre distance constraints in the range 1.5-16 nm. ${ }^{[1-6]}$ The approach is solution-based, and accesses structural and dynamic information in biomolecules such as proteins ${ }^{[7-12]}$ and nucleic acids. ${ }^{[13-}$ ${ }^{16]}$ PDEPR has contributed to conformational studies, ${ }^{[17-19]}$ disentangling competing structural models, ${ }^{[20,21]}$ and provided mechanistic insights into complex biomolecular apparatus. ${ }^{[22-24]}$ Furthermore, PDEPR has been used to monitor complexation, ${ }^{[25-27]}$ determine solution-state 
protein-ligand binding equilibria, ${ }^{[28-30]}$ and study oligomerisation-degree. ${ }^{[31-34]}$ Commonly, pairs of paramagnetic moieties, such as nitroxide radicals, are covalently introduced and conjugated with thiol side-chains of cysteine residues inserted at strategic positions via site-directed mutagenesis. ${ }^{[35,36]}$

This covalent attachment of nitroxide radicals through sulfhydryl moieties is convenient and robust $^{[35,37-39]}$, however it results in the labelling of all accessible cysteine residues and so is often intractable in systems which contain essential structural or functional cysteines. This has precipitated interest in alternative labelling chemistries and strategies, such as incorporation of genetically-encoded spin-labels as artificial amino-acids, ${ }^{[40-42]}$ and exogenously-introduced transition metal, ${ }^{[43-45]}$ or lanthanide metal centres. ${ }^{[46-49]}$ One especially promising spin-labelling approach is the co-ordination-based introduction of exogenous $\mathrm{Cu}^{\prime \prime}$-chelates at doublehistidine residue $(\mathrm{dH})$ motif sites. ${ }^{[50]}$ Typically, histidine residues are arrayed in $\alpha$-helical and $\beta$ sheet secondary structural elements, at positions $i$ to $i+4$ and $i$ to $i+2$, respectively, and $\mathrm{Cu}^{\prime \prime}$ chelated by iminodiacetic acid $\left(\mathrm{Cu}^{\prime \prime}-\text { IDA }\right)^{[51]}$ or nitrilotriacetic acid $\left(\mathrm{Cu} \mathrm{u}^{11}-\mathrm{NTA}\right)^{[52]}$ selfassemble at these sites. However, because the labelling is non-covalent, it is governed by an equilibrium determined by both the free concentrations of macromolecule and label. It was recently shown that $\mathrm{Cu}^{\prime \prime}$-NTA has low-micromolar affinities for dH sites, under cryogenic conditions. ${ }^{[28]}$

These $\mathrm{Cu}^{\prime \prime}$-chelate spin-labels also yield exquisite precision in the distance domain owing to the bipedal mode of attachment, and the rigidity of the co-ordinating imidazole moieties with respect to the protein backbone. ${ }^{[00,53]}$ The lack of a flexible linker means $\mathrm{dH} \mathrm{Cu}^{\prime \prime}$-labelling is appealing for structural studies in systems with subtle conformational changes ${ }^{[54]}$ or nuanced conformational equilibria. ${ }^{[55]}$ Furthermore, use of $\mathrm{Cu}^{\prime \prime}-N T A$ in conjunction with the commercially available methanethiosulfonate spin label MTSL, in the 5-pulse dead-time free Relaxation Induced Dipolar Modulation Enhancement (RIDME) experiment ${ }^{[56]}$ yields superb concentration sensitivity, down to hundreds of ${ }^{2 M}{ }^{[28]}$ Taken together, this makes $\mathrm{dH} \mathrm{Cu} \mathrm{Cu}^{\prime \prime}$ labelling a powerful tool for future applications in PDEPR spectroscopy.

However, considering the simple case of a tetra-histidine (double $\mathrm{dH}$ ) protein labelled with $\mathrm{Cu}^{\prime \prime}$ spin-label, optimisation of labelling efficiency for PDEPR applications can become nontrivial. ${ }^{[57]}$ Under conditions of partial loading, all $\mathrm{Cu}^{\text {Il }}$ species whether $\mathrm{dH}$ bound or not can be detected, but not all species will contribute to dipolar modulation of the detected echo. This 
has obvious implications for measurement sensitivity, being further exacerbated if each site coordinates $\mathrm{Cu}^{\prime \prime}$ spin-label with differential affinity and must therefore be treated as nonidentical and independent. To achieve widespread use of $\mathrm{dH} \mathrm{Cu}$ "labelling, there must be a means to optimize labelling efficiency in any given double $\mathrm{dH}$ system. The situation will further complicate for more than $2 \mathrm{dH}$ sites.

This problem has been approached previously using a probabilistic method to approximate

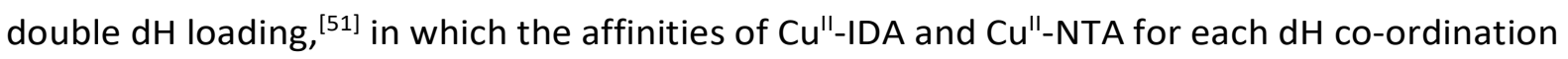
site were treated individually. However, the approximation of independent sites not depleting the free ligand concentration is not always satisfied. Thus, a model to explicitly treat two independent $\mathrm{dH}$ co-ordination sites simultaneously in a single macromolecule is currently lacking.

Here, we give a general derivation for a multi-site binding polynomial, treating a pair of binding sites with differential affinities. Numerical simulations are used to predict the optimal labelling efficiency in dependence of $K_{D}$ values, and the mathematical model is validated experimentally in a protein system. The methodology is evaluated in two aspects: i) benchmarking the theoretical treatment of modelling speciation against experiment, and ii) empirical considerations and limitations associated with the approach.

\section{Theoretical Background}

\section{Multi-site speciation model:}

Let us begin by considering the general case of a protein $(P)$ ligand $(L)$ reaction scheme, written as:

$$
P+i L \stackrel{\beta_{i}}{\leftrightarrow} P L_{i}
$$

where $\beta_{i}$ is the macroscopic association constant and is given as (square brackets giving concentrations):

$\beta_{i}=\frac{\left[P L_{i}\right]}{[P][L]^{i}}$

The binding polynomial, $Z$, can be defined as the partition function of the system, and is therefore the sum of all $n$ species with respect to the concentration of the free macromolecule, $[P]$ as a reference-state: ${ }^{[58]}$ 
$Z=\sum_{i=0}^{n} \frac{\left[P L_{i}\right]}{[P]}$

Now consider insertion of $\beta_{i}$ into the expression for $Z$ above, we have:

$Z=\sum_{i=0}^{n} \beta_{i}[L]^{i}$

For a protein system containing $s$ identical binding sites, one can convert from macroscopic association constants, $\beta_{i}$ to microscopic association constant, $K:^{[58]}$

$\beta_{i}=\left(\begin{array}{l}S \\ i\end{array}\right) K^{i}$

Therefore: in the case of $s$ identical ligand binding sites, we can define $Z$ in terms of the microscopic association constant as:

$Z=\sum_{i=0}^{S}\left(\begin{array}{l}S \\ i\end{array}\right) K^{i}[L]^{i}$

By virtue of the symmetry of Pascal's triangle of binomial coefficients this equals:

$Z=\sum_{i=0}^{s}\left(\begin{array}{l}S \\ i\end{array}\right)(K[L])^{S-i}$

Using the binomial identity, $Z$ can be defined as a $s^{\text {th }}$ order polynomial in product of free ligand concentration and microscopic association constant:

$Z=(1+K[L])^{s}$

Since $Z$ is a partition function, the fractional population of each species is given as:

$f_{i}=\frac{\left[P L_{i}\right]}{[P]_{0}}=\frac{\beta_{i}[L]^{i}}{Z}$

Consider that in the case of non-identical independent binding, that is if $K_{1} \neq K_{2}$ :

$Z=\prod_{i=1}^{n}\left(1+K_{i}[L]\right)$

We see for two-site non-identical independent binding, this gives the following definition of $Z$ :

$Z=\left(1+K_{1}[L]\right)^{s} \times\left(1+K_{2}[L]\right)^{t}$ 
where each class of sites have $s$ - and $t$-fold degeneracy, that is, $s$ and $t$ describe the number of each type of site. Then, for $s$ identical sites with microscopic association constant, $K_{1}$, and $t$ identical sites with microscopic association constant, $K_{2}$, it is known from multinomial theorem that $Z$ can be expressed as a double sum over all $i$ permutations of $s$, and $j$ permutations of $t$ :

$Z=\sum_{i=0}^{s} \sum_{j=0}^{t}\left(\begin{array}{l}S \\ i\end{array}\right)\left(K_{1}[L]\right)^{s-i}\left(\begin{array}{l}t \\ j\end{array}\right)\left(K_{2}[L]\right)^{t-j}$

This is equivalent to the form given for 2 non-identical independent binding sites. ${ }^{[58]}$ Indeed, in the case of $s$ identical sites, $t$ is 0 , so this binomial term reduces to unity, and yields the familiar expression given above in equation (8).

It should be recognised that to calculate fractional speciation via this approach, one must have a closed-form expression of the ligand concentration at equilibrium. While analytical solutions exist for polynomials with degree 4 , the corresponding roots are no longer unique, and otherwise require numerical simulation. Therefore, it is necessary to approximate all sites as belonging to two classes; considered here as high- and low-affinity, respectively. This allows calculation of $[L]$, from a polynomial with degree 3 , trigonometrically via Vietta's substitution. ${ }^{[59]}$

More explicitly, $[L]$ is cubic in $[L]_{0}$ for a two-site system, precluding cooperativity considerations. Let us derive an expression for concentration of protein-ligand complex for a one-site system:

$$
\begin{aligned}
& K_{D}=\frac{[P][L]}{[P L]} \\
& {[P]=[P]_{0}-[P L]}
\end{aligned}
$$

Substituting equation (13) into equation (12), we can write:

$K_{D}[P L]=\left([P]_{0}-[P L]\right)[L]$

Isolating total protein concentration yields:

$\frac{K_{D}[P L]+[P L][L]}{[L]}=[P]_{0}$ 
Finally, this can be rearranged to yield an expression for protein-ligand complex, as the familiar one-site Langmuir isotherm:

$\frac{[P]_{0}[L]}{K_{D}+[L]}=[P L]$

Extending this to a multi-site Langmuir isotherm yields an expression for the concentration of bound ligand, $[L]_{B}$, where from mass law, and in analogy to equation (16):

$[L]_{B}=\left([L]_{0}-[L]\right)$

Thus:

$[L]_{0}-[L]=\mathrm{s}\left(\frac{[P]_{0}[L]}{K_{D_{1}}+[L]}\right)+\mathrm{t}\left(\frac{[P]_{0}[L]}{K_{D_{2}}+[L]}\right)$

Where $\mathrm{s}$ and $\mathrm{t}$ are the number of ligand-binding sites with affinities $K_{D 1}$ and $K_{D 2}$, respectively. Equation (18) is rearranged to yield:

$\left([L]_{0}-[L]\right)\left(K_{D_{1}}+[L]\right)\left(K_{D_{2}}+[L]\right)=\left(K_{D_{2}}+[L]\right) s[P]_{0}[L]+\left(K_{D_{1}}+[L]\right) t[P]_{0}[L]$

this can be expressed as a cubic equation:

$[L]^{3}+a[L]^{2}+b[L]-c=0$

where:

$a=\left((s+t)[P]_{0}+K_{D_{1}}+K_{D_{2}}-[L]_{0}\right)$

$b=\left(t[P]_{0} K_{D_{1}}+s[P]_{0} K_{D_{2}}+K_{D_{1}} K_{D_{2}}+\left(K_{D_{1}}+K_{D_{2}}\right)[L]_{0}\right)$

$c=K_{D_{1}} K_{D_{2}}[L]_{0}$

after Vietta's substitution, the analytical expression for $[L]$ is given as:

$[L]=-\frac{a}{3}+\frac{2}{3} \sqrt{\left(a^{2}-3 b\right)} \cos \frac{\theta}{3}$

where:

$\theta=\cos ^{-1}\left(\frac{-2 a^{3}+9 a b-27 c}{2 \sqrt{\left(a^{2}-3 b\right)^{3}}}\right)$

And $a, b$, and $c$ are defined as above. 


\section{RIDME experiment}

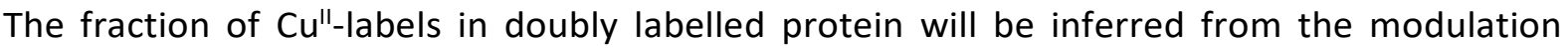
depths $(\Delta)$ of RIDME experiments. This provides a proxy for the labelling efficiency, which can be optimised by determining the maximum RIDME modulation depth as a function of total $\mathrm{Cu}^{\prime \prime}-$ label concentration.

Briefly, the 5-pulse RIDME experiment (pulse sequence in ESI) relies on intrinsic longitudinal relaxation (characterised by the phenomenological relaxation times $T_{1}$ ) of homo or hetero spin-pairs; detected $(A)$ spins are perturbed by the change in local magnetic field induced by longitudinal relaxation $\left(\Delta m_{s}\right)$ of $(B)$ spins during the interval $T_{m i x}$, and this manifests as a modulation of the detected refocused electron spin-echo by the dipolar coupling, $\omega_{A B}$. The corresponding inter-spin distance $\left(r_{A B}\right)$ is related to the frequency of this modulation ${ }^{[60,61]}$ by:

$\omega_{A B}=\frac{\mu_{0} \mu_{B}^{2}}{8 \pi^{2} \hbar} \frac{g_{A} g_{B}}{r_{A B}^{3}}\left(1-3 \cos ^{2} \theta_{A B}\right)$

Where $\mu_{0}$ is the vacuum permeability constant, $\mu_{B}$ is the Bohr magneton, $\hbar$ is the reduced Planck constant, $g_{A}$ and $g_{B}$ are respective $g$-values of each spin, $r_{A B}$ is the inter-spin distance and $\vartheta_{A B}$ is the angle between the inter-spin vector and the external magnetic field vector.

Modulation depth build-up depends on both the length of the interval $T_{m i x}$, and $T_{1}$. Under the approximation of mono-exponential $T_{1}$ behaviour, the asymptotic modulation depth $\left(\Delta_{\text {Tmix }}\right)$ for a given ratio of $T_{\text {mix }}$ and $T_{1}$, is given as:

$\Delta_{\text {Tmix }}=\frac{\left(1-\exp \left(\frac{-T_{\text {mix }}}{T_{1}}\right)\right)}{2}$

It should be recognised that in the limiting case where: $\Delta=\Delta_{T m i x}$, the fraction of bound Cull-

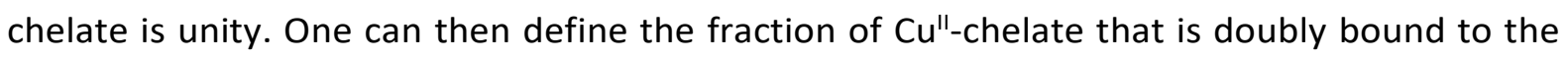
macromolecule $\left(Q_{\text {exp }}\right)$, given as:

$\Delta \times \Delta_{\text {Tmix }}{ }^{-1}=Q_{\text {exp }}$

This equivalence is used interchangeably from this point onwards. 


\section{Experimental Procedures}

\section{Protein purification and EPR sample preparation:}

All Streptococcus $s p$. group G protein G, B1 domain (GB1) protein constructs $(16 \mathrm{H} / \mathrm{N} 8 \mathrm{H} / \mathrm{K} 28 \mathrm{H} / \mathrm{Q} 32 \mathrm{H}, 16 \mathrm{R} 1 / \mathrm{K} 28 \mathrm{H} / \mathrm{Q} 32 \mathrm{H}$ and $16 \mathrm{H} / \mathrm{N} 8 \mathrm{H} / \mathrm{K} 28 \mathrm{R} 1)$ were produced, expressed, purified and spin labelled as previously reported. ${ }^{[28,50]} \mathrm{Cu}^{\prime \prime}$-chelate spin labels were prepared and quantified as previously reported. ${ }^{[28,62]}$ In all samples, addition of $50 \%(\mathrm{v} / \mathrm{v})$ ethylene glycol (EG) $d-6$ (Deutero $\mathrm{GmbH}$ ) ensured formation of a glassy frozen solution, after addition of $\mathrm{Cu}^{\prime \prime}$ nitrilotriacetic acid (Cu"-NTA) label, to a total volume of $70 \mu \mathrm{L}$, unless otherwise stated. All EPR samples were immediately flash-frozen in $\mathrm{N}_{2}($ () by direct immersion, following preparation.

\section{EPR instrumentation:}

All pulse EPR experiments were performed using a Bruker ELEXSYS 580 pulse EPR spectrometer. Temperatures were maintained using a cryogen-free variable temperature cryostat (Cryogenic Ltd) operating in the 1.8-300 K temperature range. All measurements of the electron spin longitudinal relaxation times $\left(T_{1}\right)$, and transverse dephasing times $\left(T_{m}\right)$ of $\mathrm{Cu}^{\prime \prime}$ IDA and Cu"-NTA, and all 5-pulse dead-time free RIDME measurements ${ }^{[56]}$ were performed at $30 \mathrm{~K}$, using a high-power $150 \mathrm{~W}$ travelling-wave tube (TWT; Applied Systems Engineering) at Q-band $(34 \mathrm{GHz})$ in a critically coupled $3 \mathrm{~mm}$ cylindrical resonator (Bruker ER 5106QT-2w in TE012 mode). All 4-pulse dead-time free Pulse Electron-electron Double Resonance (PELDOR) measurements ${ }^{[63]}$ were performed at $10 \mathrm{~K}$ at X-band $(9.4 \mathrm{GHz}$ ) with a $1 \mathrm{~kW}$ TWT (Applied Systems Engineering) in an over-coupled $3 \mathrm{~mm}$ split-ring resonator (Bruker 4118X-MS3), unless otherwise stated.

\section{Pulse EPR spectroscopy measurement parameters:}

The 5-pulse dead-time free RIDME experiment ( $\left.\pi / 2-\tau_{1}-\pi-\tau_{1}-t-\pi / 2-T_{\text {mix }}-\pi / 2-\left(\tau_{2}-t\right)-\pi-\tau_{2}-e c h o\right)$ was used (see ESI) with rectangular pulses of lengths 12 and 24 ns ( $\pi / 2$ and $\pi$, respectively), consistently placing the detection frequency to the maximum of the Cull-NTA spectrum. All acquisitions were performed over three scans (unless otherwise stated), with 10 shots per point, with a shot repetition time (SRT) of $2 \mathrm{~ms}$, and deuterium ESEEM was suppressed via a 16-step nuclear modulation $\tau$-averaging cycle. ${ }^{[64]}$ Signal contributions from unwanted echoes were eliminated using an 8-step phase-cycle, totalling 128 steps per measurement (resulting in 384 echoes per point per scan), with the refocused virtual echo (RVE) being detected. For all samples, at least two lengths of mixing block were recorded; a short reference mixing time 
( $\left.T_{\text {ref }}\right)$ and a long mixing time $\left(T_{\text {mix }}\right)$ to allow suppression and observation of the dipolar coupling, respectively. A pseudo-titration series (a titration with discrete samples prepared for each data point in the series) of $16 \mathrm{H} / \mathrm{N} 8 \mathrm{H} / \mathrm{K} 28 \mathrm{H} / \mathrm{Q} 32 \mathrm{H}$ GB1 was measured at $100 \mu \mathrm{M}$ protein concentration, in presence of varying $\mathrm{Cu}^{\prime \prime}$-NTA concentration. All RIDME data was used without division by the reference trace and background corrected using stretched exponential functions with stretching exponent bounded between 1 and 2, unless otherwise stated (see $E S I)$.

The 2-pulse electron spin-echo decay (ESE) experiment $(\pi / 2-\tau-\pi)$ was applied at the maximum field position of the Cu"-chelate spectrum (see ESI), using 16 and $32 \mathrm{~ns} \pi / 2$ - and $\pi$-pulses. Traces were acquired with a trace length of 20 or $33 \mu$ s as stated, measured at $30 \mathrm{~K}$, using a $\tau$ of 800 ns (due to increased dead-time in high Q mode), which was incremented in steps of 8 ns, for 4096 points. For all measurements, an SRT of 2 ms was used. Raw data were fitted using a stretched-exponential function to estimate $T_{m}$ :

$y=A_{0}\left[\exp \left(\left(\frac{-\tau}{T_{m}}\right)^{x}\right)\right]$

The 3-pulse inversion recovery (IR) experiment $(\pi-T-\pi / 2-\tau-\pi)$ was used and applied at the maximum field position of the $\mathrm{Cu}^{\prime \prime}$-chelate spectrum with a $12 \mathrm{~ns}$ inversion pulse (nominal flipangle $\pi$ ). 20 and 40 ns pulses ( $\pi / 2$ - and $\pi$, respectively) were used for the observer subsequence (unless otherwise stated). Traces were acquired to $500 \mu$ s and the time interval $T$ was incremented in steps of $200 \mathrm{~ns}$, using an SRT of $2 \mathrm{~ms}$ (unless otherwise stated) and a $\tau$ of 800 ns. Raw data were fitted with mono- and bi-exponential functions, to estimate $T_{1}$ :

$y=A_{0}\left[1-2 a \times \exp \left(\frac{-x}{T_{1}}\right)\right]$

$y=A_{0}\left[1-2 a\left(b \times \exp \left(\frac{-x}{T_{1 B \text { slow }}}\right)+(1-b) \times \exp \left(\frac{-x}{T_{1 B \text { fast }}}\right)\right)\right]$

For the Cu"-detected X-band PELDOR measurements, samples of 250 and $220 \mu \mathrm{M}$ $16 \mathrm{R} 1 / \mathrm{K} 28 \mathrm{H} / \mathrm{Q} 32 \mathrm{H}$ and $16 \mathrm{H} / \mathrm{N} 8 \mathrm{H} / \mathrm{K} 28 \mathrm{R} 1 \mathrm{~GB} 1$, respectively, were measured either in presence of 1.5 equivalents of $C u^{\prime \prime}-$ IDA or $C u^{\prime \prime}-N T A$. The 4-pulse experiment $\left(\pi / 2\left(U_{A}\right)-\tau_{1}-\pi\left(U_{A}\right)-\tau_{1}-t-\pi\left(U_{B}\right)-\left(\tau_{2^{-}}\right.\right.$ t)- $\pi\left(U_{A}\right)-t_{2}$-echo) was used, where $U_{A}$ and $U_{B}$ indicate the pulse excitation at the observer and pump frequencies, respectively. In all cases monochromatic pulses of lengths 16 and 32 and 12 ns were used for observer and pump pulses $(\pi / 2, \pi$ and $\pi)$. The magnetic field and microwave frequency were adjusted to the maximum of the nitroxide spectrum to coincide 
with the pump pulse position, while the observer pulse spectral position was varied between frequency offsets of 150 and $300 \mathrm{MHz}$ depending on the measurement. Data were acquired with an SRT of $3 \mathrm{~ms}$, a $\tau_{1}$ of $420 \mathrm{~ns}$ and a $\tau_{2}$ of $1260 \mathrm{~ns}$ were used respectively, with 50 shotsper-point and measurements averaged for 12 hours to yield sufficient signal-to-noise ratio (SNR). ${ }^{2} \mathrm{H}$ ESEEM was suppressed using a 16 -step t-averaging cycle.

\section{5-pulse RIDME data processing, analysis and validations:}

All 5-pulse RIDME traces were processed using DeerAnalysis2018, and were backgroundcorrected using either second order polynomial or stretched-exponential functions as specified in the text, and the observed modulation depth quotients were found to be robust against background correction models (see ESI). The stretched exponential functions were of the form: ${ }^{[65]}$

$$
y=c \times \exp \left(-(k \times t)^{\frac{d}{3}}\right)
$$

Where $c$ is the initial amplitude, $k$ is the decay constant, $t$ is the time-point, and $d$ is the background dimension, which is constrained between 3 and 6 , in keeping with recent RIDME theory. ${ }^{[66]}$ For the validations of the RIDME traces (see ESI), a modified version of the Tikhonov validation functionality in DeerAnalysis $2018^{[67]}$ was used; a total of 896 trials were performed for each validation, consisting of 16 white-noise iterations (noise-level of 1.5), 8 iterations of background start position (between $5-30 \%$ of the total RIDME trace length), and 7 iterations of background dimension (between 3-6 in increments of 0.5 ). From each pruned validation trial (prune level $=1.15$ ) a modulation depth $(\Delta)$ is calculated, and the standard deviation $(\sigma)$ is used to approximate the modulation depth error, relevant in the propagation of errors to calculate $K_{D}$ from RIDME pseudo-titration series (see ESI). Bivariate fitting used in-house Matlab scripts and employed a Nelder-Mead simplex. Each $K_{D}$ value was varied independently, and a least-squares bivariate error minimisation was performed (see ESI). The root-mean square deviation (RMSD) was used as an estimate of goodness-of-fit between simulation and experiment. Fits were found to be largely stable regardless of chosen initial parameters. 


\section{Results and Discussion}

\section{Simulation of $16 \mathrm{H} / \mathrm{N} 8 \mathrm{H} / \mathrm{K} 28 \mathrm{H} / \mathrm{Q} 32 \mathrm{H}$ GB1 speciation and modulation depth profile:}

Since the case of a $\mathrm{Cu}$ "l homo spin-pair $(S=1 / 2)$ is discussed, all spins will contribute to the detected echo signal. This means the detected echo will be the weighted contributions of unbound, singly-bound, and doubly-bound (macromolecules with both dH-motifs occupied) $\mathrm{Cu}^{\prime \prime}$ spin-label. However, only the doubly-bound macromolecule will modulate the detected echo signal with the dipolar frequency; therefore, the observed modulation depth is the relative quotient of $\mathrm{Cu}^{\prime \prime}$ spins which are doubly-bound, against all $\mathrm{Cu}^{\prime \prime}$ spins present in the sample, $[L]_{0}$. This can be succinctly expressed as:

$Q_{\text {exp }}=\frac{[L]_{1,2}}{[L]_{0}}$

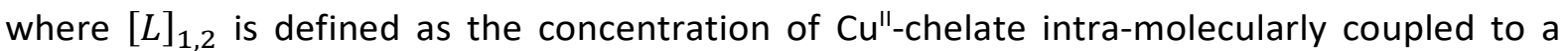

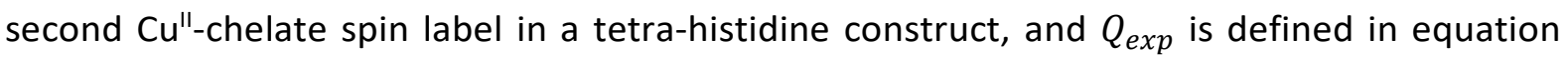
(28). Therefore, the modulation depth profile is a log-normal function with increasing $[L]_{0}$. The definition of $[L]_{1,2}$ is given as:

$[L]_{1,2}=2 \theta_{1,2}[P]_{0}$

where $\theta_{1,2}$ is defined as: ${ }^{[68,69]}$

$\theta_{1,2}=\frac{\left(K_{1} K_{2} K_{12}\right)[L]^{2}}{Z}$ 
a)

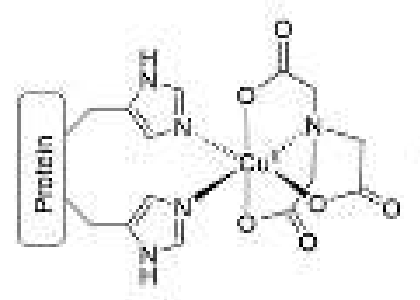

c)

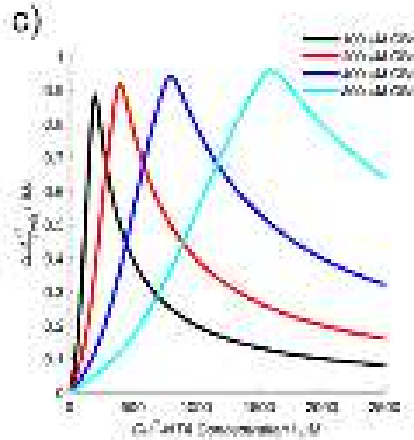

b)

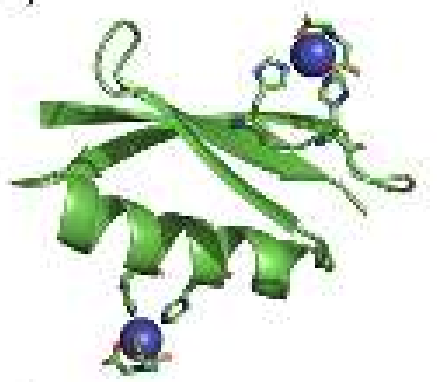

d)

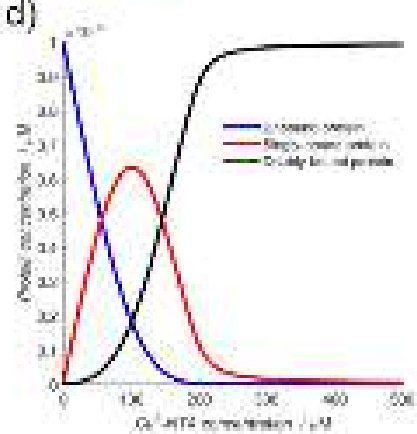

Figure 1. a) The structure of the Cull-NTA spin label, coordinated to the $\delta$-nitrogen atoms of the imidazole rings of a protein $\mathrm{dH}$ site. b) Double $\mathrm{dH}(16 \mathrm{H} / \mathrm{N} 8 \mathrm{H} / \mathrm{K} 28 \mathrm{H} / \mathrm{Q} 32 \mathrm{H} \mathrm{GB1})$ construct in cartoon representation (PDB: 4WH4), [16] with the $\mathrm{Cu}^{\prime \prime}$-NTA spin labels and co-ordinating $\mathrm{dH}$ sites in stick representation and $\mathrm{Cu}^{\prime \prime}$ ions as blue spheres. c) A simulation of modulation depth quotient as a function of increasing Cu"l-NTA concentration for 100 (black), 200 (red), 400 (blue) and 800 (cyan) $\mu \mathrm{M}$ 16H/N8H/K28H/Q32H GB1. d) A simulated speciation plot for $100 \mu \mathrm{M}$ protein showing the concentrations of unbound (blue), singly bound (red), and doubly bound (black) protein as a function of Cu"-NTA concentration. The axis is truncated to $500 \mu \mathrm{M}$, to better resolve each curve. Panels a) and b) are taken from figure 1 of reference 28 .

where $K_{12}$ describes the cooperativity of the binding mode, which here is assumed to be noncooperative $\left(K_{12}=1\right)$. Therefore, using the model elaborated in the theory section, a modulation depth profile is simulated for various concentrations of $16 \mathrm{H} / \mathrm{N} 8 \mathrm{H} / \mathrm{K} 28 \mathrm{H} / \mathrm{Q} 32 \mathrm{H}$ GB1

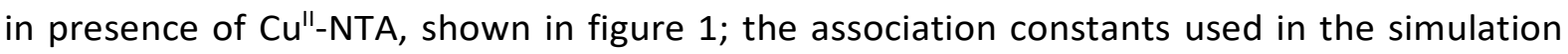
$\left(K_{1}=7.14 \times 10^{6}\right.$ and $\left.K_{2}=7.14 \times 10^{5}\right)$ are estimates from previous work. ${ }^{[28]}$ Panels c $)$ and d) show that $\mathrm{dH}$ loading is essentially quantitative under these simulated conditions, and a sensitivity optimum is anticipated at approximately a protein-to-Cu"-NTA ratio of 1:2.

The log-normal shape of the modulation depth profiles with increasing Cu"-NTA concentration is significant. The implication is that for a double $\mathrm{dH}$ construct, the sensitivity optimum is a point-solution on the curve, rather than a plateau, observed as for the Cu"-nitroxide case, which yields a hyperbolic single site saturation function. ${ }^{[28]}$ The 'breadth' of this optimum is determined by both $K_{D}$ values, and the protein concentration; at higher protein concentrations, for a fixed $K_{D}$ pair, the profile decays to the right of the maximum less steeply, 
and shifts the maximum towards unity (see figure $1 \mathrm{c}$ ). The sensitivity optimum will tend towards unity for increasing protein concentrations, for any value of $K_{D}$. However, in practice, it may be more useful to measure at lower protein concentration, while maintaining high sensitivity in a concentration regime several orders of magnitude greater than the predicted $K_{D}$ values (see ESI).
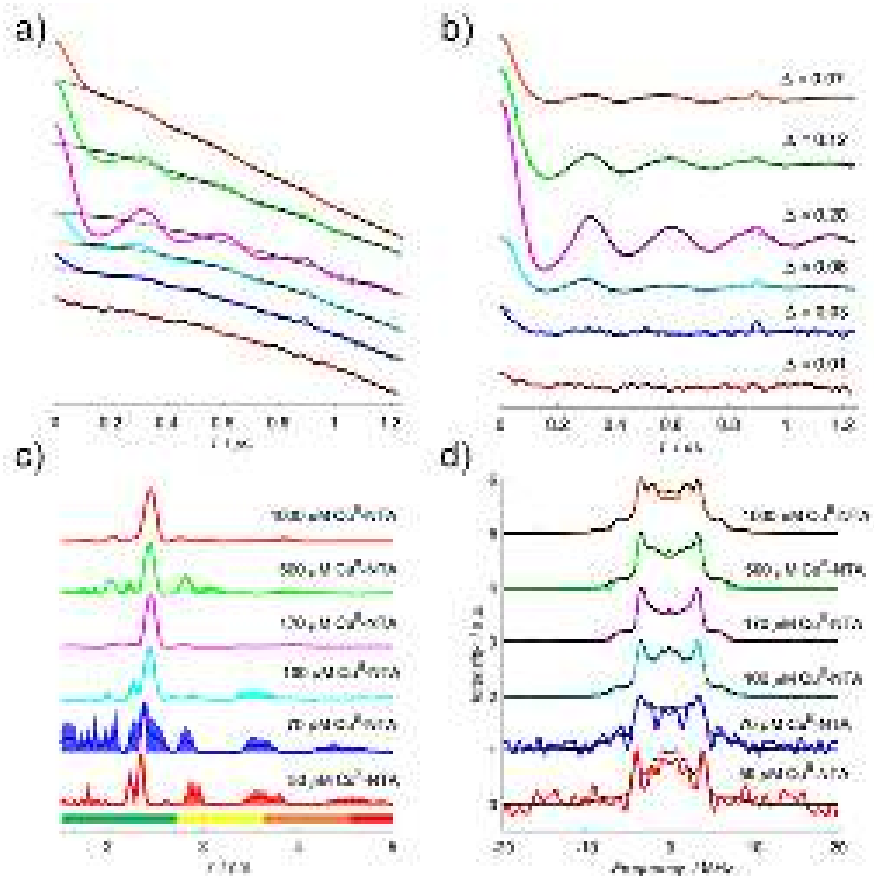

Figure 2. a) A stack-plot of the raw RIDME traces for $100 \mu \mathrm{M} \mathrm{6H/8H/28H/32H} \mathrm{GB1}$ in presence of 50 (red), 70 (blue), 100 (cyan), 170 (magenta), 500 (green) and $1000 \mu \mathrm{M} \mathrm{Cu}$-NTA (orange), recorded using a ratio of $T_{\text {mix }}$ to $T_{1}$ of 0.7 . Stretched exponential background functions are shown as black dotted lines. Data have been shifted vertically for visibility. b) A stack plot of the background corrected data from a) with their fits shown as black dotted lines. c) The corresponding distance distributions calculated from the data in $\mathrm{b}$ ) and offset vertically to aide visualisation. The $2 \mathrm{~s}$ confidence intervals are shown as the shaded background for each distribution. d) The dipolar spectra corresponding to the data in b), each is offset vertically to aide visualisation.

\section{Double-dH pseudo-titration with Cu"-NTA:}

To investigate the validity and robustness of the speciation model developed above, a pseudotitration series of $100 \mu \mathrm{M} 16 \mathrm{H} / \mathrm{N} 8 \mathrm{H} / \mathrm{K} 28 \mathrm{H} / \mathrm{Q} 32 \mathrm{H}$ GB1 in presence of 50, 70, 100, 170, 500 and $1000 \mu \mathrm{M}$ Cu"-NTA was prepared. For a homo-spin pair there is a trade-off between measurement sensitivity and the accuracy of the observed modulation depth quotients, $\left(Q_{\text {exp }}\right)$, depending on the error in the approximated $T_{1}$ value. Importantly, the largest source of error will likely manifest as deviations from the mono-exponential approximation, shown in equation (30). RIDME measurements were performed with 3 experimental mixing times, since 
the observed modulation depth is a function of both $\mathrm{dH}$ loading and $\Delta_{T m i x}$. Mixing times between 0.7 and $1.9 \times T_{1}$ were used to determine the consistency of the modulation depth quotients.

The RIDME data recorded with a ratio of $\sim 0.7$ between mixing time and $T_{1}$ are shown in figure 2. Quantitatively, the trend in modulation depth is consistent with expectation. This is best seen in panel b) upon comparison of the empirical modulation depths which first increase towards $20 \%$ before reducing as excess Cu"-NTA is added. Panel c) of figure 2 demonstrates that at low $\mathrm{dH} \mathrm{Cu}^{\prime \prime}$-labelling, the reliability of the distance distributions is substantially reduced compared to optimal labelling conditions. Nevertheless, reliable modulation depth information could still be extracted (see ESI). This further emphasizes the utility of being able to identify optimal labelling conditions for a given system, in the purview of extracting reliable, meaningful distances for Cu"-Cu" RIDME.

The dipolar spectra are shown in panel d), the sample measured in presence of $170 \mu \mathrm{M} \mathrm{Cu}$ "NTA gives a spectrum closely resembling a Pake pattern and indicating minimal effects from orientational correlation. However, other points in the series yield spectra with low signal-tonoise (as seen for the 50 and $70 \mu \mathrm{M}$ Cu"-NTA samples) or with additional singularities (as seen for the $1000 \mu \mathrm{M}$ Cu"-NTA sample). An advantage of the 5-pulse RIDME experiment is a reduced susceptibility to orientation selection arising from broadband B-spin excitation only limited by relaxation anisotropy rather than pulse excitation bandwidth as in PELDOR. ${ }^{[70-72]}$ Orientation selection has previously been demonstrated to be significant for double $\mathrm{dH} \mathrm{Cu}^{\prime \prime}-\mathrm{Cu}$ " PELDOR at Q-band frequencies. ${ }^{[73]}$

It was observed that the raw 5-pulse RIDME traces contained an additional feature at $~ 900 \mathrm{~ns}$ in the dipolar evolution functions; it is most prominent in the 70 and $100 \mu \mathrm{M} C \mathrm{Cu}^{\prime \prime}-\mathrm{NTA}$ samples but persists to varying degrees in all cases. This was attributed to a standing echo artefact that can likely be suppressed through use of an extended 32-step phase-cycle. ${ }^{[65]}$ Here, an 8-step phase-cycle was retained for the sake of simplicity as the presence of the artefact did not affect the downstream data analysis. For further discussion and measurements performed using the extended phase-cycle see ESI. Initial concern that for low signal-to-noise ratio samples, the presence of the artefact would artificially inflate the white-noise estimation in the error analysis did not manifest (see ESI). 


\section{Bivariate fitting of dissociation constants}

The pseudo-titration series (treated with a stretched exponential background function) for all ratios of $T_{\text {mix }}$ and $T_{1}$ are shown in figure 3 , and pairs of dissociation constants are fitted to experimental modulation depth quotients $\left(Q_{\text {exp }}\right)$, according to equation (36).

$\operatorname{rmsd}=\operatorname{mean}\left(\left(Q_{\text {sim }}-Q_{\text {exp }}\right)^{2}\right)$

Where $Q_{\text {sim }}$ can be calculated in dependence of a pair of dissociation constants from equations (27, 33-35).

Importantly, the fit demonstrates that both sites differ by an order of magnitude in affinity, in good agreement with data from Cu"-NTA nitroxide RIDME pseudo-titrations in previous work (simulated here as black dotted trace in figure 3). ${ }^{[28]}$ The fitted dissociation constants are given in the figure legend. The determined affinities from the $\mathrm{Cu}^{\prime \prime}-\mathrm{Cu}$ " RIDME pseudo-titration are comparable to the affinities derived from previous Cu"-nitroxide RIDME pseudo-titrations for each of the double histidine motifs individually. In the previous study, affinities were determined for the $\alpha$-helical $28 \mathrm{H} / 32 \mathrm{H}$ site $(140 \mathrm{nM})$ and the $\beta$-sheet $6 \mathrm{H} / 8 \mathrm{H}$ site $(1.4 \mu \mathrm{M})$, respectively.

The error associated with modulation depth quotients quantified via $\mathrm{Cu}^{\text {"-}}-\mathrm{Cu}$ " RIDME will tend to be larger than those quantified via $\mathrm{Cu}^{\prime \prime}$-nitroxide RIDME. This is because, as mentioned above there is a trade-off between sensitivity and modulation-depth quotient accuracy, as the mixing time increases. To investigate this systematically, error analyses for all Cu"-Cu" RIDME pseudo-titration series (with ratios of $T_{\text {mix }}$ to $T_{1}$ of approximately $0.7,1.3$ and 1.9, respectively)

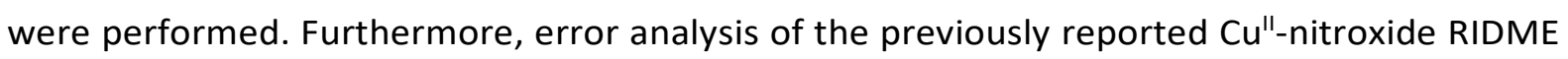
pseudo-titration series was performed, for comparison (see ESI). The $95 \%$ confidence intervals in observed modulation depths $(\Delta)$ did not exceed \pm 0.03 of the distribution means for all cases, comparable to the previous $\mathrm{Cu}^{\prime \prime}$-nitroxide series. 

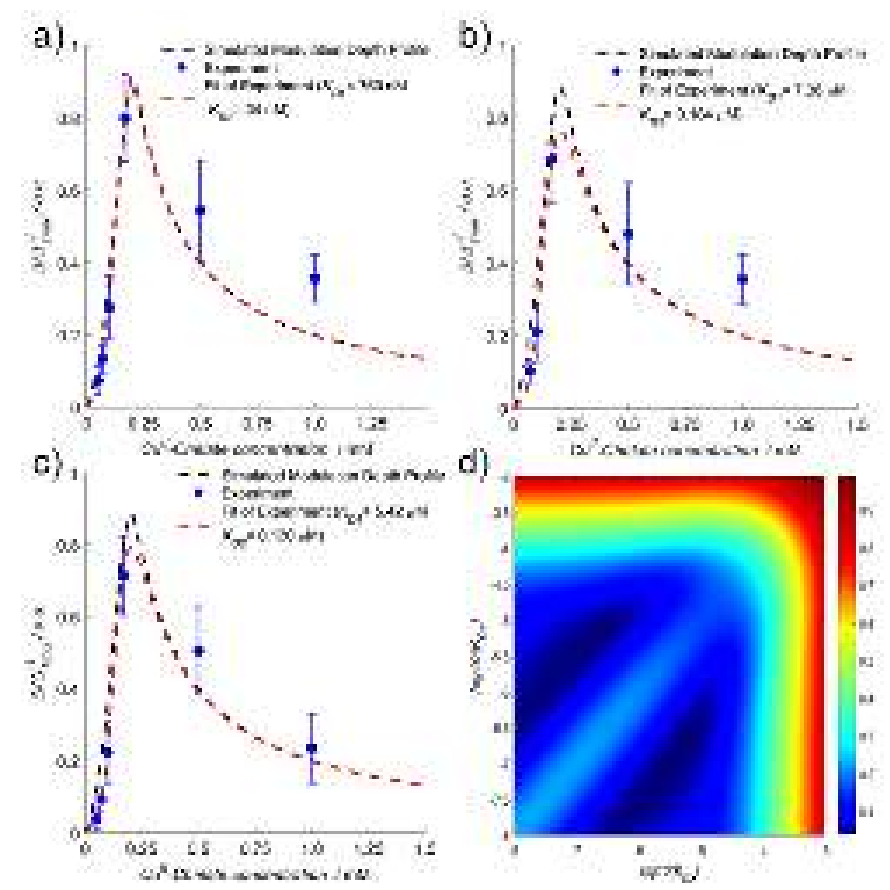

Figure 3. The experimental modulation depth quotients calculated using a stretched exponential background function (blue scatter), overlaid with the predicted modulation depth profile (black dashes) and the associated bivariate fit (red dashes) recorded with a mixing time of a) $0.7 \times T_{1}$, b) $1.3 \times T_{1}$, and $1.9 \times T_{1}$. d) An error surface of the bivariate fitting of each dissociation constant to the experimental data shown in panels a-c). The colour bar indicates the normalised RMSD. Each dissociation constant varies 5 orders of magnitude from $10 \mathrm{nM}$ to $1 \mathrm{mM}$.

The relative error in the modulation depth quotients $\left(Q_{\text {exp }}\right)$ generally reduces at longer mixing time and was typically constrained to $\pm 25 \%$ for a $T_{\text {mix }}$ and $T_{1}$ ratio of 1.9. Results are summarised in the ESI, for both the stretched exponential and second-order polynomial background correction models. Figure 3 shows that there is general agreement between the empirical modulation depth quotients and the fit, regardless of the $T_{\text {mix }}$ and $T_{1}$ ratio used. However, it is apparent that only for the series recorded with the highest ratio do all experimental data points lie on the fitted curve (within error), and the original simulated modulation depth profile. A potential point of interest is that for all ratios of $T_{m i x}$ and $T_{1}$, the second order polynomial marginally outperformed the stretched exponential background correction model. While the manifest differences are small in terms of the fitted $K_{D}$ values, modulation depth quotients derived from the second order polynomial corrected datasets were more robust against changes in the $T_{\text {mix }}$ and $T_{1}$ ratio (see ESI).

Importantly, all data shown in figure 3 were also fitted globally (for all ratios of $T_{\text {mix }}$ and $T_{1}$ ) and $K_{D}$ values were similar to estimates from the fits shown in figure 3, particularly for the $T_{\text {mix }}$ to $T_{1}$ ratio of 1.9 (see ESI). Differences in the affinity estimates are reasonably small for all ratios 
of $T_{\text {mix }}$ and $T_{1}$, and pseudo-titrations recorded at all ratios of $T_{\text {mix }}$ and $T_{1}$ reproduce the order of magnitude difference between the differential $\mathrm{dH}$ sites. Sampling several $T_{\text {mix }}$ and $T_{1}$ ratios and fitting all data together also seems to improve the accuracy of the fitted $K_{D}$ values. See below for further discussion.

The consistency of dissociation constants determined via both Cu"-nitroxide and Cu"-Cu" RIDME pseudo-titrations is encouraging and suggests that accurate information regarding binding equilibria can be extracted from double $\mathrm{dH}$ systems, in a single measurement series. However, where available $\mathrm{Cu}^{\prime \prime}$-nitroxide and $\mathrm{Cu}^{\prime \prime}-\mathrm{Cu}^{\prime \prime} \mathrm{RIDME}$ pseudo-titrations can be combined, to independently validate binding affinities. A global fitting approach of both $\mathrm{Cu}^{\prime \prime}-$ $\mathrm{Cu}^{\prime \prime}$ and $\mathrm{Cu}^{\prime \prime}$-nitroxide RIDME shows a slight improvement of the fit (see ESI). It should also be noted that $\mathrm{Cu}^{\prime \prime}-\mathrm{Cu}$ " RIDME pseudo-titrations are likely to be of greater diagnostic value in systems where binding sites differ by an order of magnitude or greater in their respective affinities.

Interestingly, the error surface (Figure 3 ) reveals that the two dissociation constants are positively correlated, evidenced by the symmetry about the leading diagonal. This is likely because the shape of the modulation depth profile is highly sensitive to the magnitude of the individual $K_{D}$ values, and not simply their product. Further, the error surface demonstrates that the $K_{D}$ values are distinct, owing to the ridge along the leading diagonal. Indeed, simulation with $K_{D}$ values of i) $100 \mathrm{nM}$ and $1 \mu \mathrm{M}$, and ii) $10 \mathrm{nM}$ and $10 \mu \mathrm{M}$ (where individual $K_{D}$ values are increased and decreased respectively, by an order of magnitude, but their product remains unchanged) show that the agreement between simulation and experiment is poorer in the latter case, particularly at lower Cu"-NTA concentrations, and the maximum modulation depth quotient reduces from $\sim 0.9$ to $<0.75$.(see ESI).

However, it should be appreciated that the profile sensitivity to a given pair of $K_{D}$ values is also determined by the total protein concentration. For instance, in the limiting case of high affinity the simulated profiles are largely identical, and the discerning feature becomes the maximum modulation depth quotient (see ESI). In this case, moving into a lower protein concentration regime would be desirable, and would improve sensitivity of $K_{D}$ determination. Measurements at protein concentrations in the same range as the $K_{D}$ value afford higher measurement accuracy, ${ }^{[74-76]}$ however owing to sensitivity limitations associated with detection of small 
modulation depth changes in intentionally under-labelled samples, an empirical protein concentration of $100 \mu \mathrm{M}$ was chosen.

The observation that the $K_{D}$ values are not 'compensatory' has important implications for the robustness of the model. It allows one to 'compartmentalise' the profile into the initial flank, maximum and the region to the right of the maximum, in discussions regarding the higher-and lower-affinity $K_{D}$ values. Since the region to the right of the maximum is independent of either $K_{D}$ and only dependent upon the ratio of double $\mathrm{dH}$ protein and total $\mathrm{Cu}^{\prime \prime}$ chelate concentrations, it can be used as an internal control, to assess the concentration accuracy of the pseudo-titration series. This is discussed in more detail in the ESI.

\section{Considerations for Accurate Cu"-Cu" RIDME Modulation Depth Quantitation:}

One consideration of using a coordination-based spin labelling method, is that relaxation behaviour may differ between the free and bound components. This means that under conditions of partial loading, the relative contributions of free and $\mathrm{dH}$-bound $\mathrm{Cu}^{\text {"l-chelate to }}$ the detected echo will be different. To demonstrate this, inversion recovery, and 2-pulse electron-spin echo decay traces for quantitatively free and dH-bound Cu"-IDA and Cu"-NTA were recorded (see ESI). Free $\mathrm{Cu}^{\prime \prime}$-chelate has a slower rate of transverse relaxation $\left(T_{m}\right)$, and a faster rate of longitudinal relaxation $\left(T_{1}\right)$, compared to $\mathrm{dH}$-bound $\mathrm{Cu}$ "-chelate. This supports previous results that suggested the $T_{1}$ increases with increasing $\mathrm{dH}$-binding, regardless of relative concentration; an effect observed across concentrations ranging 5 orders of magnitude.

In this work, Cu"-Cu" RIDME modulation depths were approximated to be independent of transverse relaxation. Importantly, this is justified by considering that RIDME experiments were performed using a total dipolar evolution time of $3.8 \mu \mathrm{s}$, and this time window is sufficiently short to approximate the transverse relaxation behaviour of free and $\mathrm{dH}$-bound $\mathrm{Cu}^{\prime \prime}$ to be largely identical. However, it is doubtful this approximation applies to all dipolar evolution times. Perhaps more significant is the longitudinal relaxation behaviour, ${ }^{[25]}$ since a fast $T_{1}$ component can cause modulation depths at shorter mixing times to be overrepresentative of the extent of $\mathrm{dH}$-binding. This is partially addressed here by using several mixing times of varying length with respect to $T_{1}$, although sensitivity becomes limiting at sufficiently long mixing time intervals. Nevertheless, for all mixing times the trend in $\Delta$ was found to be consistent, and no further treatment was required. 
It was also observed that for Cu"-nitroxide PELDOR measurements, the modulation depth varied when selecting different spectral positions corresponding to the $\mathrm{Cu}^{\prime \prime}$-chelate for the echo forming pulses. This is attributed to different spectra and maxima of the two species. Modulation depths were lower when detection was performed at higher offset. Previous literature values ${ }^{[77]}$ and simulation of free and $\mathrm{dH}$-bound $\mathrm{Cu}^{\prime \prime}$-chelate using Easyspin ${ }^{[78]}$, indicated $\mathrm{g}_{\|}$is smaller for the $\mathrm{dH}$-bound, relative to the free component, resulting in greater excitation at lower offset, consistent with the observed trend (see ESI). Since Cu"-Cu" RIDME

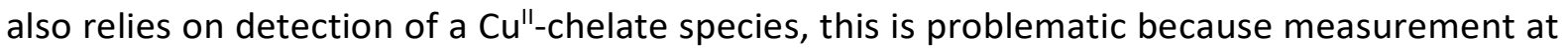
two distinct field positions would yield different affinity estimates, and therefore not be robust. However, it should be noted that all RIDME measurements in this work were performed using the maximum of the $\mathrm{Cu}^{\prime \prime}$-chelate spectrum as the detection position, which would commonly be the most desirable position (ignoring effects from angular correlations and orientation selection) to ensure a high SNR. Furthermore, we find that the affinities estimated from the $\mathrm{Cu}^{\text {"I }}-\mathrm{Cu}$ " RIDME measurements closely align with previous estimates stated

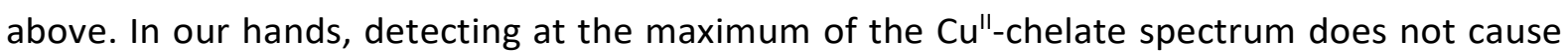
significant deviations in the apparent $K_{D}$, however this is not necessarily satisfied for all field positions.

Results in this work suggest that when accurate modulation depth quantitation is paramount, the axiom of maximising sensitivity for a homo-spin pair in 5-pulse RIDME by using $0.7 \times T_{1}$ as $T_{\text {mix }}$ may not be optimal. However, perhaps the furthest reaching implication of determining $K_{D}$ via pulse EPR, is that it allows the coupling of structural and binding equilibria information. Therefore, the aim should be to find a compromise wherein both modulation depth information and structural information can be reliably extracted. In practice, using $0.7 \times T_{1}$ as $T_{\text {mix }}$ gives a good first impression of binding equilibria information in the system under investigation. Even so, a systematic treatment of how to optimise modulation depth quantitation and $K_{D}$ determination from $\mathrm{Cu}^{\prime \prime}$-nitroxide and $\mathrm{Cu}^{\prime \prime}-\mathrm{Cu}^{\prime \prime}$ 5-pulse RIDME pseudotitrations is necessary. Importantly, robust modulation depth quantitation can serve as an internal control of the labelling efficiency; to the right of the sensitivity maximum, additional ligand will be unbound, and thus dilute the bound component which contributes to the modulation.

\section{Conclusions}


Our findings demonstrate that modulation depth quantitation in a $\mathrm{Cu}^{\prime \prime}$ homo-spin system via 5-pulse RIDME is feasible; binding equilibria information can be reliably obtained, and empirical observation agrees nicely with theoretical prediction from the general multi-site binding model developed herein. Results indicate that the previously benchmarked method of $K_{D}$ determination via 5-pulse RIDME can be extended to an analytical two-site independent binding model. Potential empirical considerations for modulation depth quantitation in double $\mathrm{dH}$ constructs have also been discussed.

One exciting prospect is that PDEPR allows intimate coupling of both structural and thermodynamic information. Furthermore, the sensitivity of PDEPR is significantly higher than for other techniques used to study thermodynamic parameters, such as isothermal titration calorimetry (ITC), and so could be used in concert with these techniques. It is also recognised that through orthogonal labelling routines or using spectroscopically orthogonal spin centres, it may become feasible to investigate complicated protein-ligand binding equilibria via modulation depth quantitation between different subsets of spins, simultaneously. This is particularly appealing in comparison to ITC since calorimetric methods have historically struggled to disentangle non-specific or multiple sequential binding events.

Additionally, the results presented herein also showcase that dissociation constants can be investigated and derived for systems which are not amenable to site-directed spin labelling with an organic radical spin label. This is significant because it expands the utility of this approach to proteins containing essential cysteine residues. The further confirmation of high $\mathrm{nM}$ and low $\mu \mathrm{M}$ affinities of $\alpha$-helical and $\beta$-sheet $\mathrm{dH}$ motif sites for Cu"-NTA, respectively, holds promise for their future widespread application in the field of PDEPR. Finally, the mathematical model derived above can be appropriated to solve sensitivity optima and maximise labelling efficiency for coordination-based spin labelling strategies, governed by binding equilibria, and may be especially useful in cases where binding affinity is limiting.

\section{Conflicts of interest}

There are no conflicts to declare.

\section{Acknowledgements}

The authors thank Dr Hassane El Mkami for help with EPR. JLW is supported by the BBSRC DTP Eastbio. This work was supported by equipment funding through the Wellcome Trust (099149/Z/12/Z) and 
BBSRC (BB/R013780/1). We gratefully acknowledge ISSF support to the University of St Andrews from the Wellcome Trust.

\section{Notes and references}

1 A. D. Milov, K. M. Salikhov, and M. D. Schirov, Fiz. Tverd. Tela., 1981, 23, 975.

2 A. D. Milov, A. B. Ponomarev, and Y. D. Tsvetkov, Chem. Phys. Lett., 1984, 110, 67.

3 T. Schmidt, M. A. Walti, J. L. Baber, E. J. Hustedt, and G. M. Clore, Angew. Chem. Int. Ed., 2016, 55, 15905.

4 G. Jeschke, Emerg. Top. Life. Sci., 2018, 2, 9.

5 H. Sanabria, D. Rodnin, K. Hemmen, T. Peulen, S. Felekyan, M. R. Fleissner, M. Dimura, F. Koberling, R. Kuhnemuth, W. Hubbel, H, Gohlke, C. A. M Seidel, Nat. Commun., 2020, 11, 1231.

6 O. Duss, M. Yulikov, F. H. T. Allain, G. Jeschke, Meth. Enzymol., 2015, 558, 279.

7 E. H. Yardeni, T. Bahrenberg, R. A. Stein, S. Mishra, E. Zomot, B. Graham, K. L. Tuck, T. Huber, E. Bibi, H. S. Mchaourab, and D. Goldfarb, Sci. Rep., 2019, 9, 12528;

8 T. Strohaker, B. C. Jung, S. Liou, C. O. Fernandez, D. Riedel, S. Becker, G. M. Halliday, M. Bennati, W. S. Kim, S. Lee, and M. Zweckstetter, Nat. Commun., 2019, 10, 5535.

9 S. Chuo, S. Liou, L. Wang, R. D. Britt, T. L. Poulos, I. F. Sevrioukova, and D. B. Goodin, Biochemistry, 2019, 58, 3903.

10 M. Kim, S. A. Vishivetskiy, N. V. Eps, N. S. Alexander, W. M. Cleghorn, X. Zhan, S. M. Hanson, T. Morizumi, O. P. Ernst, J. Meiler, V. V. Gurevich, and W. L. Hubbell, Proc. Natl. Acad. Sci. USA., 2012, 109, 18407.

11 D. T. Edwards, T. Huber, S. Hussain, K. M. Stone, M. Kinnebrew, I. Kaminker, E. Matalon, M. S. Sherwin, D. Goldfarb, and S. Han, Structure, 2014, 22, 1677.

12 B. Verhalen, R. Dastvan, S. Thangapandian, Y. Peskova, H. A. Koteiche, R. K. Nakamoto, E. Tajkho and H. S. Mchaourab, Nature, 543, 738.

13 O. Schiemann, N. Piton, Y. Mu, G. Stock, J. W. Engels, and T. F. Prisner, J. Am. Chem. Soc., 2004, 126, 5722.

14 C. Wuebben, S. Blume, D. Abdullin, D. Brajtenbach, F. Haege, S. Kath-Schorr, and O. Schiemann, Molecules, 2019, 24, 4482.

15 O. Duss, M. Yulikov, G, Jeschke, and F. H. T. Allain, Nat. Commun. 2014, 5, 3669.

16 E. S. Babaylova, A. A. Malygin, Alexander A. Lomzov, D. V. Pyshnyi, M. Yulikov, G. Jeschke, O. A. Krumkacheva, M. V. Fedin, G. G. Karpova, and E. G. Bagryanskaya, Nucleic Acids Res. 2016, 44, 7935. 
17 H. Sameach, A. Narunsky, S. Azoulay-Ginsberg, L. Gevorkyan-Aiapetov, Y. Zehavi, Y. Moskovitz, T. Juven-Gershon, N. Ben-Tal, and S. Ruthstein, Structure, 2017, 25, 988.

18 A. Dalaloyan, A. Martorana, Y. Barak, D. Gataulin, E. Reuveny, A. Howe, M. Elbaum, S. Albeck, T. Unger, V. Frydman, E. H. Abdelkader, G. Otting, and D. Goldfarb, Chemphyschem., 2019, 20, 1860.

19 B. Joseph, A. Sikora, and D. S. Cafiso, J. Am. Chem. Soc. 2016, 138, 1844.

20 F. X. Theillet, A. Binolfi, B. Bekei, A. Martorana, H. M. Rose, M. Stuiver, S. Versini, D. Lorenz, M. van Rossum, D. Goldfarb, and P. Selenko, Nature, 2016, 530, 45.

21 P. S. Kerry, H. L. Turkington, K. Ackermann, S. A. Jamieson, and B. E. Bode, J. Phys. Chem. B., 2014, 118, 10882.

22 C. A. J. Hutter, M. Hadi-Timachi, L. M. Hurlimann, I. Zimmerman, P. Egloff, H. Goddeke, S. Kucher, S. Stefanic, M. Kartunnen, L. V. Schafer, E. Bordignon and M. A. Seeger, Nat. Commun., 2019, 10, 2260.

23 C. Kapsalis, B. Wang, H. El Mkami, S. J. Pitt, J. R. Schnell, T. K. Smith, J. D. Lippiat, B. E. Bode, and C. Pliotas, Nat. Commun., 2019, 10, 4619.

24 D. Constantinescu-Aruxandei, B. Petrovic-Stojanovska, O. Schiemann, J. H. Naismith, and M. F. White, Nucleic Acids Res., 2016, 44, 954.

25 A. Giannoulis, M. Oranges, and B. E. Bode, Chemphyschem., 2017, 18, 2318.

26 K. Ackermann, A. Giannoulis, D. B. Cordes, A. M. Z. Slawin and B. E. Bode, Chem. Commun., 2015, 51, 5257.

27 A. Giannoulis, K. Ackermann, P. Spindler, C. Higgins, D. B. Cordes, A. M. Z. Slawin, T. F. Prisner, and B. E. Bode, Phys. Chem. Chem. Phys., 2018, 20, 11196.

28 J. L. Wort, K. Ackermann, A. Giannoulis, A. J. Stewart, D. G. Norman, and B. E. Bode, Angew. Chem. Int. Ed., 2019, 58, 11681.

29 J. Glaenzer, M. F. Peter, G. H. Thomas, and G. Hagelueken, Biophys. J., 2017, 112, 109.

30 A. Collauto, H. A. DeBerg, R. Kaufmann, W. N. Zagotta, S. Stoll, and D. Goldfarb, Phys. Chem. Chem. Phys., 2017, 19, 15324.

31 E. A. Riederer, P. J. Focke, E. R. Georgieva, N. Akyuz, K. Matulef, P. P. Borbat, J. H. Freed, S. C. Blanchard, O. Boudker, and F. I. Valiyaveetil, eLife., 2018, 7, e36478.

32 A. Giannoulis, R. Ward, E. Branigan, J. H. Naismith, and B. E. Bode, Mol. Phys. 2013, 111, 2845.

33 G. Hagelueken, W. J. Ingledew. H. Huang, B. Petrovic-Stojanovska, C. Whitfield, H. El Mkami, O. Schiemann, and J. H. Naismith, Angew. Chem. Int. Ed., 2009, 48, 2904.

34 T. Schmidt, R. Ghirlando, J. Barber, and G. M. Clore, Chemphyschem. 2016, 17, 2987.

35 W. L. Hubbell, and C. Altenbach, Curr. Opin. Struct. Biol., 1994, 4, 566. 
36 T. Braun, M. Drescher, and D. Summerer, Int. J. Mol. Sci. 2019, 20, 373.

37 S. Kim, Y. Jang, S. Ha, J. Ahn, E. Kim, J. H. Lim, C. Cho, Y. S. Ryu, S. K. Lee, S. Y. Lee, and K. Kim, Nat. Commun., 2015, 6, 8410.

38 P.Khanal, Z. Jia, and X. Yang, Sci. Rep., 2018, 8, 3485.

39 D. W. Bak, M. D. Pizzagalli, and E. Weerapana, ACS Chem. Biol., 2017, 12, 947.

40 G. E. Merz, P. P. Borbat, A. R. Muok, M. Srivastava, D. N. Bunck, J. H. Freed, and B. R. Crane, J. Phys. Chem. B., 2018, 122, 9443.

41 E. G. B. Evans, and G. L. Millhauser, Meth. Enzymol., 2015, 563, 503.

42 M. J. Schmidt, J. Borbas, M. Drescher, and D. Summerer, J. Am. Chem. Soc., 2014, 136, 1238.

43 E. Narr, A. Godt, and G. Jeschke, Angew. Chem. Int. Ed., 2002, 114, 4063.

44 B. E. Bode, J. Plackmeyer, T. F. Prisner, and O. Schiemann, J. Phys. Chem. A., 2008, 112, 5064.

45 Z. Wu, A. Feintuch, A. Collauto, L. A. Adams, L. Aurelio, B. Graham, G. Otting, and D. Goldfarb, J. Phys. Chem. Lett., 2017, 8, 5277.

46 L. Garbuio, E. Bordignon, E. K. Brooks, W. L. Hubbell, G. Jeschke and M. Yulikov, J. Phys. Chem. B., 2013, 117, 3145.

47 A. Shah, A. Roux, M. Starck, J. A. Mosely, M. Stevens, D. G. Norman, R. I. Hunter, H. El Mkami, G. Smith, D. Parker, and J. E. Lovett, Inorg. Chem., 2019, 58, 3015.

48 G. Prokopiou, M. D. Lee, A. Collauto, E. H. Abdelkader, T. Bahrenberg, A. Feintuch, M. Ramirez-Cohen, J. Clayton, J. D. Swarbrick, B. Graham, G. Otting, and D. Goldfarb, Inorg. Chem., 2018, 57, 5048.

49 D. Barthelmes, M. Granz, K. Barthelmes, K. N. Allen, B. Imperiali, T. F. Prisner, and H. Schwalbe, J. Biomol. NMR. 2015, 63, 275.

50 T. F. Cunningham, M. R. Putterman, A. Desai, W. S. Horne, and S. Saxena, Angew. Chem. Int. Ed., 2015, 54, 6330.

51 M. J. Lawless, S. Ghosh, T. F. Cunningham, A. Shimshi, and S. Saxena, Phys. Chem. Chem. Phys., 2017, 19, 20959.

52 S. Ghosh, M. J. Lawless, G. S. Rule, and S. Saxena, J. Magn. Reson., 2018, 286, 163.

53 S. Ghosh, S. Saxena, and G. Jeschke, Appl. Magn. Reson., 2018, 49, 1281.

54 H. Sameach, S. Ghosh, L. Gevorkyan-Airapetov, S. Saxena, and S. Ruthstein, Angew. Chem. Int. Ed., 2019, 10, 3053.

55 M. J. Lawless, J. R. Petterson, G. S. Rule, F. Lanni, and Sunil Saxena, Biophys. J., 2018, 114, 592.

56 S. Milikisyants, F. Scarpelli, M. G. Finiguerra, M. Ubbink, and M. Huber, J. Magn. Reson., 2009, 201, 48.

57 A. Gamble Jarvi, J. Casto, and S. Saxena, J. Magn. Reson., 2020, 320, 106848. 
58 E. Freire, A. Schön, and A. Velazquez-Campoy, Meth. Enzymol., 2009, 455, 127.

59 Z. X. Wang, and R. F. Jiang, FEBS Lett., 1996, 392, 245.

60 A. D. Milov, A. G. Marysov, and Y. D. Tsvetkov, Appl. Magn. Reson., 1998, 15, 107.

61 S. Valera, and B. E. Bode, Molecules. 2014, 19, 20227.

62 J. Gao, F. Xing, Y. Bai, and S. Zhu, Dalton Trans., 2014, 43, 7964.

63 M. Pannier, S. Veit, A. Godt, G. Jeschke, and H. W. Spiess, J. Magn. Reson., 2000, 142, 331.

64 K. Keller, A. Doll, M. Qi, A. Godt, G. Jeschke, and M. Yulikov, J. Magn. Reson., 2016, 272, 108.

65 I. Ritsch, H. Hintz, G. Jeschke, A. Godt and M. Yulikov, Phys. Chem. Chem. Phys., 2019, 21, 9810.

66 K. Keller, Q. Mian, C. Gmeiner, I. Ritsch, A. Godt, G. Jeschke, A. Savitsky, and M. Yulikov, Phys. Chem. Chem. Phys., 2019, 21, 8228.

67 S. G. Worswick, J. A. Spencer, G. Jeschke, and I. Kuprov, Sci. Adv., 2018, 4, 5218.

68 J. J. Wyman, Adv. Protein Chem., 1964, 19, 223.

69 D. F. Senear, and M. Brenowitz, J. Biol. Chem., 1991, 266, 13661.

70 D. Abdullin, F. Duthie, A. Meyer, E. S. Muller, G. Hagelueken, and O. Schiemann, J. Phys. Chem. B., 2015, 119, 13534.

71 D. Abdullin, H. Matsuoka, M. Yulikov, N. Fleck, C. Klein, S. Spicher, G. Hagelueken, S. Grimme, A. Lutzen, and O. Schiemann, Chem. Eur. J., 2019, 25, 8820.

72 S. Razzaghi, M. Qi, A. I. Nalepa, A. Godt, G. Jeschke, A. Savitsky, and M. Yulikov, J. Phys. Chem. Lett., 2014, 5, 3970.

73 A. G. Jarvi, K. Ranguelova, S. Ghosh, R. T. Weber, and S. Saxena, J. Phys. Chem. B., 2018, 122, 10669.

74 J. R. Horn, D. Russell, E. A. Lewis, and K. P. Murphy, Biochemistry., 2001, 40, 1774.

75 L. Baranauskiene, V. Petrikaite, J. Matuliene, and D. Matulis, Int. J. Mol. Sci., 2009, 10, 2752.

76 S. A. Kantonen, N. M. Henriksen, and M. K. Gilson, Biochim. Biophys. Acta. Gen. Subj., 2017, 1861, 485.

77 K. Singewald, X. Bogetti, K. Sinha, G. S. Rule, S. Saxena, Angew. Chem. Int. Ed., 2020, DOI: https://doi.org/10.1002/anie.202009982.

78 S. Stoll, A. Schweiger, J. Magn. Reson., 2006, 178, 42. 